\title{
Stereoselective synthesis of tetrahydrofuranyl 1,2,3-triazolyl $C$ - nucleoside analogues by 'click' chemistry and investigation of their biological activity
}

\author{
P. Venkat Reddy, ${ }^{\text {a }}$ Mohammad Saquib, ${ }^{\text {a }}$ Nripendra N. Mishra, \\ Praveen K. Shukla ${ }^{b}$ and Arun K. Shaw ${ }^{\mathrm{a} *}$ \\ ${ }^{a}$ Division of Medicinal and Process Chemistry, CSIR-Central Drug Research Institute, Lucknow \\ 226031, India \\ ${ }^{b}$ Medical Mycology Lab, Division of Fermentation Technology, CSIR-Central Drug Research \\ Institute, Lucknow 226031, India \\ E-mail:akshaw55@yahoo.com
}

DOI: http://dx.doi.org/10.3998/ark.5550190.p008.374

\begin{abstract}
The construction of a novel series of enantiopure tetrahydrofuranyl 1,2,3-triazolyl $C$-nucleoside analogues utilizing click reaction of sugar derived tetrahydrofuranyl alkynes with various "in house' synthesized sugar derived tetrahydrofuranyl azides and an adamantyl azide is described. The biological significance of the synthesized $C$-nucleosides was highlighted by evaluating them in vitro for anti-bacterial and anti-fungal activity, wherein a number of compounds were found to show excellent anti-bacterial activity and moderate anti-fungal activity.
\end{abstract}

Keywords: THF alkynes, THF azides, $C$-nucleosides, 'click' chemistry, 1,2,3-triazoles

\section{Introduction}

$C$-Nucleosides have been recognized as important compounds in the field of medicinal chemistry and chemical biology. ${ }^{1,2}$ These are unique class of nucleosides in which the heterocycle is connected to the sugar moiety with a stable $\mathrm{C}-\mathrm{C}$ bond instead of labile nucleosidic $\mathrm{C}-\mathrm{N}$ bond. The strong $\mathrm{C}-\mathrm{C}$ bond increases the stability of the glycosidic bond. The naturally occurring as well as synthetic $C$-nucleosides are known for their interesting biological properties like antibiotic, antiviral and antineoplastic (Figure1). ${ }^{3-12}$ Owing to their structural relationship to the naturally occurring nucleosides the $C$-nucleosides can be incorporated into DNA/RNA instead of the naturally occurring nucleosides. ${ }^{12}$

1,2,3-Triazoles have emerged as an important pharmacophore ${ }^{13-14}$ showing diverse type of bioactivities. ${ }^{15-20}$ In this context the importance of 1,2,3-triazole nucleosides exhibiting anti-viral, 
immunosuppressant and anti-tumor cell proliferation activities have been well documented in literature. ${ }^{21-25}$ Recently a number of 1,2,3-triazolyl carba-nucleosides have also been reported as anti-viral agents. ${ }^{26-28}$ A literature survey revealed that 1,2,3- triazole $C$-nucleosides have been less intensively studied than the other classes of 1,2,3-triazole nucleosides. Only few reports are available on synthesis of 1,2,3- triazole C-nucleosides. In 1980s M. A. E. Sallam and coworkers ${ }^{29-34}$ and later on some other research groups have reported the synthesis of 1,2,3-triazole $C$-nucleosides ${ }^{35}$ including biologically relevant molecules like 1,2,3- triazolyl analogues of ribavirin, ${ }^{36}$ an anti-viral drug and other $C$-nucleosides like ethynyl-bridged $C$-nucleoside. ${ }^{37}$

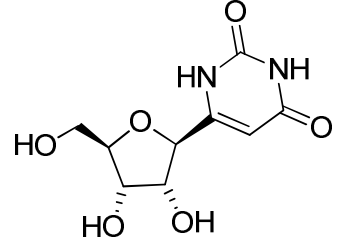

Pseudouridine (1)

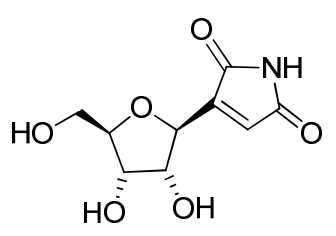

Showdomycin (3)

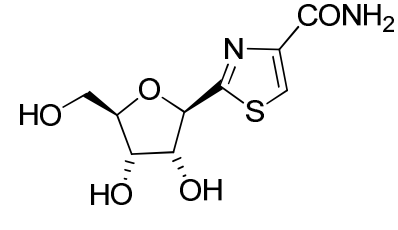

Tiazofurin (2)



Pyrazofurin (4)

Figure 1. Some biologically important $C$-nucleosides.

The interesting biological activities exhibited by 1,2,3-triazole containing compounds, specifically 1,2,3- triazole $C$-nucleosides coupled with the biological importance of $C$ nucleosides prompted us to design and synthesize stereochemically pure tetrahydrofuran (THF)alkynes and polyynes derived novel tetrahydrofuranyl 1,2,3-triazolyl $C$-nucleoside analogues and screen them for biological activity.

Huisgen 1,3-dipolar cycloaddition between an alkyne and azide is an important reaction for the preparation of 1,2,3-triazole derivatives. ${ }^{38-43}$ The development of its very efficient copper catalyzed click reaction has made this reaction the most widely used method for the synthesis of 1,2,3-triazole. ${ }^{39,40}$ Consequently in our present study we chose the copper catalyzed click reaction for the synthesis of the targeted 1,2,3- triazolyl $C$-nucleoside analogues of prototype-I and prototype-II (Figure 2). 




Figure 2. Prototype tetrahydrofuranyl 1,2,3-triazolyl C-nucleoside analogue molecules.

\section{Results and Discussion}

Retrosynthetic strategy for the construction of 1,2,3-triazolyl C-nucleoside analogues is summarized in figure 3 . The synthetic route depicted in figure 3 allowed us to prepare several 1,2,3-triazolyl $C$-nucleoside analogues differing in the relative stereochemical configuration at C-2, C-3, and C-4 positions of the THF ring. The THF domain 6 was easily obtained in three steps from 3,4,6-tri- $O$-benzyl-D-glucal or 3,4,6-tri- $O$-benzyl-D-galactal following our previously published procedure which were in turn synthesized from D-glucose and D-galactose respectively. ${ }^{44,45}$

The domain 6 was then subjected to oxidative cleavage with $\mathrm{H}_{5} \mathrm{IO}_{6}$ followed by treatment with Bestmann-Ohira reagent (dimethyl-1-diazo-2-oxopropylphosphonate) to yield the THF alkynes of the general formula $7 .^{46}$ These alkynes were reacted with different THF azides, $\mathbf{9 - 1 1}{ }^{47}$ and an adamanty azide (Figure 4) to obtain the target tetrahydrofuranyl 1,2,3-triazolyl Cnucleoside analogues of general formula $\mathbf{8}$.

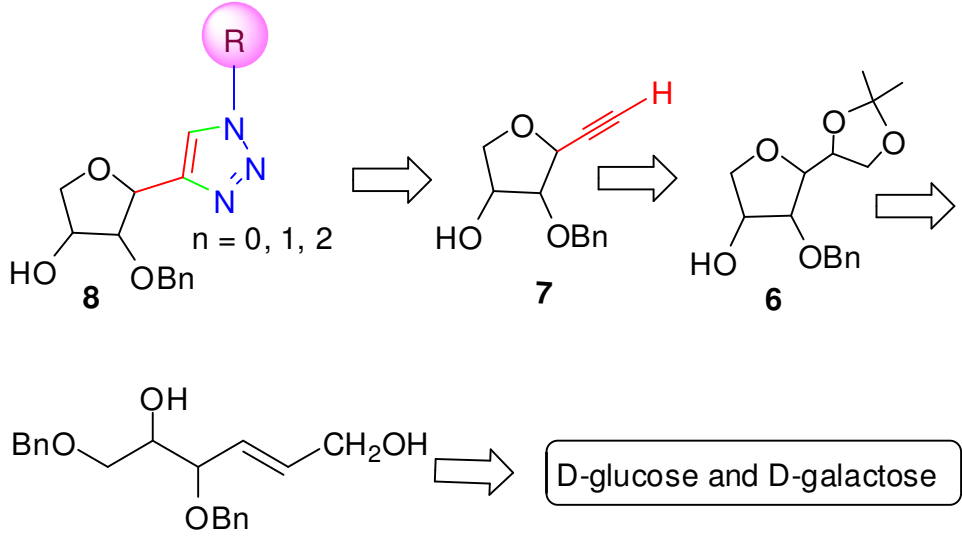

5

Figure 3. Retrosynthetic strategy for the construction of 1,2,3- triazolyl $C$-nucleoside analogues. 
The treatment of alkyne 13 with THF azide 9 in the presence of $\mathrm{CuSO}_{4} .5 \mathrm{H}_{2} \mathrm{O},(+)$-sodium Lascorbate and t-BuOH$-\mathrm{H}_{2} \mathrm{O}(1: 1)$, at ambient temperature furnished the desired triazolyl $C$ nucleoside analogue 14 in $62 \%$ yield (Scheme 1).

The structure of triazolyl $C$-nucleoside analogue 14 was established by its NMR and mass spectroscopic data. Its ${ }^{1} \mathrm{H}$ NMR spectrum showed a singlet at $\delta 7.44$ corresponding to the triazolyl proton while the ${ }^{13} \mathrm{C}$ NMR spectrum showed peaks at $\delta 121.5$ and 147.8 corresponding to $\mathrm{CH}$ and $\mathrm{qC}$ characteristic to the triazole core unit. Finally the ESI-MS spectrum displayed the $[\mathrm{M}+\mathrm{H}]^{+}$peak at $m / z 538$ and its HRMS spectrum showed the $[\mathrm{M}]^{+}$peak at 537.2490, which confirmed its structure.

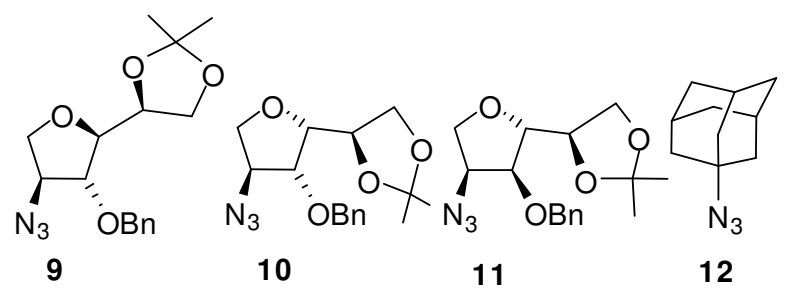

Figure 4. Azides used in the present study.

Once the synthesis of triazolyl C-nucleoside $\mathbf{1 4}$ has been successfully achieved we extended the synthetic strategy to access more compounds of this series. Different stereoisomers of THF alkynes and azides were prepared and coupled with each other to obtain a series of isomeric 1,2,3-triazolyl $C$-nucleoside analogues. Consequently when the stereoisomeric alkynes 13, 17 and 21 were treated with THF azides 9-11 under Huisgen 1,3-dipolar cycloaddition reaction condition, the corresponding tetrahydrofuranyl 1,2,3-triazolyl $C$-nucleosides analogues 15, $\mathbf{1 8}$, 19, 22, 23 and 24 were obtained in 58\% - 75\% yields (Scheme 1).

In recent years adamantane containing molecules have received much attention in medicinal chemistry. ${ }^{48-51}$ Adamantyl compounds are currently in clinical use for the treatment of neurogenerative diseases, influenza, type II diabetes and acne vulgaris. ${ }^{49}$ Many adamantane derivatives have been reported to show anti-bacterial and anti-fungal activities. ${ }^{50,51}$ The above reports encouraged us to carry out the synthesis of adamantane containing 1,2,3-triazolyl $C$ nucleosides analogues. Consequently on treating alkynes 13, 17, 21, 26 with adamantyl azide 12, adamantyl substituted triazolyl C-nucleoside analogues 16, 20, 25 and 27 respectively were obtained in $70-78 \%$ yields (Scheme 1).

Interestingly in the case of alkyne 17 derived triazoles 18, 19 and 20 the peak characteristic of $\mathrm{CH}$ and $\mathrm{q} C$ of the triazole was inexplicably recorded as a very weak signal in ${ }^{13} \mathrm{C} \mathrm{NMR}$ spectrum, but their ${ }^{1} \mathrm{H}$ NMR, ESI mass and HRMS spectroscopic studies clearly showed that the desired triazoles were formed. The structures of the triazoles $\mathbf{1 8}, \mathbf{1 9}$ and $\mathbf{2 0}$ were finally confirmed by recording their HSQC spectra which showed a correlation between carbon signals at 120.4 ppm in case of compounds $\mathbf{1 8}, \mathbf{1 9}$, and $122.4 \mathrm{ppm}$ in compound $\mathbf{2 0}$ with their respective triazole $\mathrm{CH}$ protons. 

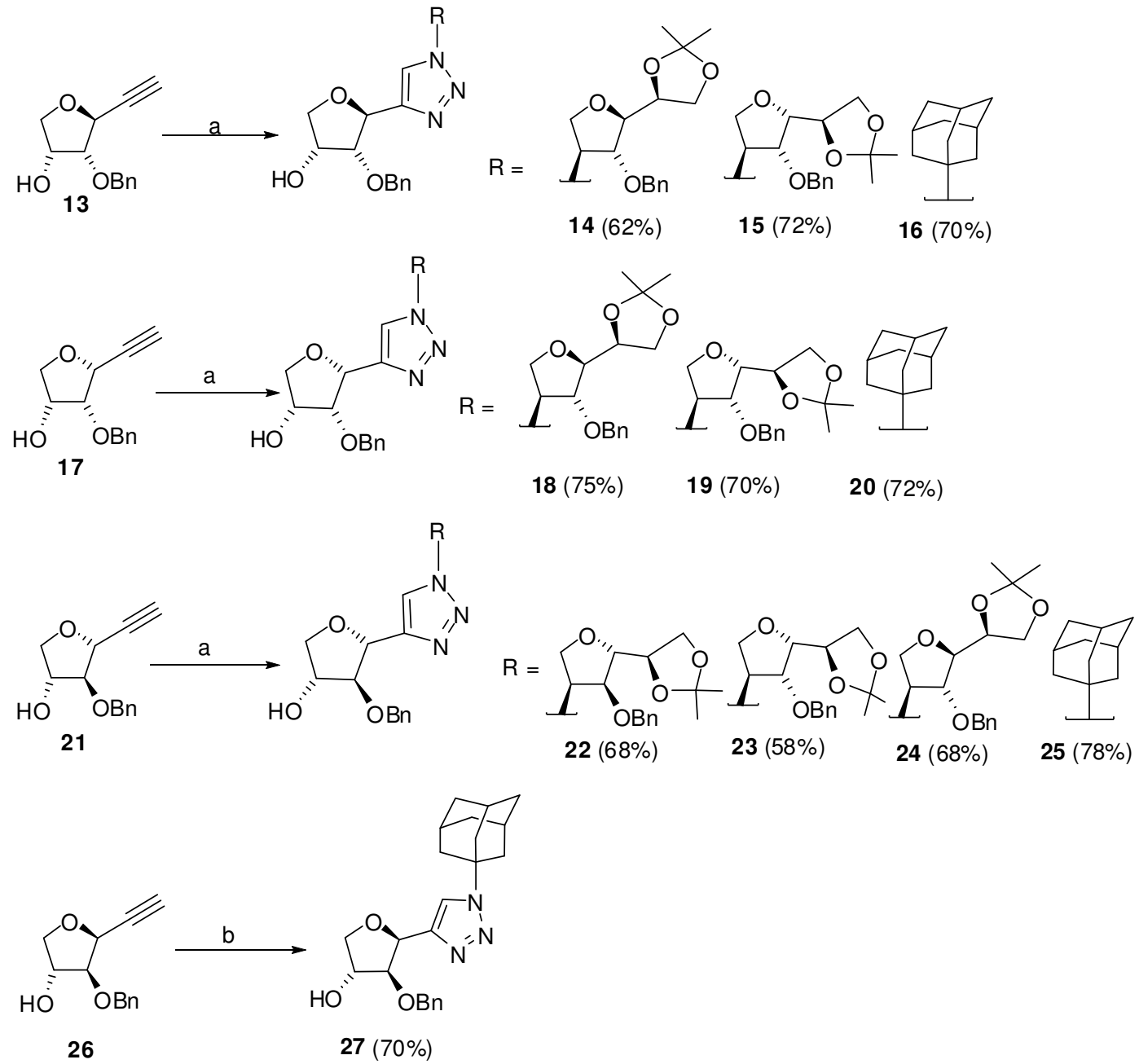

Scheme 1. Reagents and condition: (a) $\mathrm{R}-\mathrm{N}_{3}, \mathrm{CuSO}_{4} .5 \mathrm{H}_{2} \mathrm{O}$, (+)-Sodium L-ascorbate, t-BuOH$\mathrm{H}_{2} \mathrm{O}$ (1:1), rt, 6-8 h. (b) Adamantyl azide 12, $\mathrm{CuSO}_{4} .5 \mathrm{H}_{2} \mathrm{O}$, (+)-Sodium L-ascorbate, t-BuOH$\mathrm{H}_{2} \mathrm{O}(1: 1), \mathrm{rt}, 8 \mathrm{~h}$

All the above described tetrahydrofuranyl 1,2,3-triazoly C-nucleoside analogues were evaluated for their in vitro anti-bacterial and anti-fungal activities (See Table 1 and 2 in SI). It was found that the adamantane containing 1,2,3-triazolyl $C$-nucleoside analogue 25 was active at a MIC of $1.56 \mu \mathrm{g} / \mathrm{ml}$ against $S$. aureus which is two fold better activity than standard drug gentamycin and three fold better activity than ampicillin while in case of $K$. pneumoniae it exhibited a MIC of $0.78 \mu \mathrm{g} / \mathrm{ml}$ which is equivalent to the activity of gentamycin. Another adamantane containing analogue 27 was found active at a MIC of $0.78 \mu \mathrm{g} / \mathrm{ml}$ against $S$. aureus which is three fold better activity than gentamycin, four fold better activity than ampicillin and equal to ciprofloxacin. In case of $K$. pneumoniae it again exhibited a MIC of $0.78 \mu \mathrm{g} / \mathrm{ml}$ which is equivalent to the activity of gentamycin. However, none of the other 1,2,3-triazolyl C-nucleoside analogues were found to show any anti-bacterial activity. 
The 1,2,3-triazoly $C$-nucleoside analogues were also screened against a panel of six fungal strains. Only molecules 16, 18, 19 and 20 were found active against $T$. mentagrophytes at MIC of $25 \mu \mathrm{g} / \mathrm{ml}, 12.5 \mu \mathrm{g} / \mathrm{ml}, 25 \mu \mathrm{g} / \mathrm{ml}$ and $25 \mu \mathrm{g} / \mathrm{ml}$ respectively. None of the other molecules showed any activity against the fungal strains.

\section{Conclusions}

In summary we have disclosed the stereoselective construction of a novel class of 1,2,3-triazolyl $C$-nucleoside analogues (14-16, 18-20, 22-25 and 27), with multiple points for skeletal diversification, from 'THF alkynes and THF azides, using 'click' chemistry as the key step. The alkynes and azides used in this study were easily accessed from commercially available sugars. In order to demonstrate the potential biological utility of the synthesized 1,2,3-triazolyl $C$ nucleoside analogues, they were evaluated for in vitro anti-bacterial and anti-fungal activity. It was found that the adamantane containing 1,2,3-triazolyl $C$-nucleoside analogues 25 and 27 exhibited excellent activity against $S$. aureus and $K$. pneumoniae in a MIC range of $0.78 \mu \mathrm{g} / \mathrm{ml}$ to $1.56 \mu \mathrm{g} / \mathrm{ml}$ which was comparable or better than the activity of many standard drugs while compounds 16, 18, 19 and 20 exhibited moderate anti-fungal activity in a MIC range of 12.5 $\mu \mathrm{g} / \mathrm{ml}$ to $25 \mu \mathrm{g} / \mathrm{ml}$. A detailed study on further elaboration of these 1,2,3-triazolyl $C$-nucleoside analogues and investigation of their prospective bioactivities is currently underway and would be disclosed in due course.

\section{Supporting Information}

Copies of ${ }^{1} \mathrm{H},{ }^{13} \mathrm{C}$ and 2D NMR spectral data of all the synthesized 1,2,3-triazolyl $C$-nucleoside analogues, general procedure for in vitro anti-bacterial and anti-fungal activity evaluation and tabulated results of in vitro anti-bacterial and anti-fungal activity evaluation of the 1,2,3-triazolyl $C$-nucleoside analogues.

\section{Experimental Section}

General. Organic solvents were dried by standard methods. Analytical TLC was performed using $2.5 \times 5 \mathrm{~cm}$ plates coated with a $0.25 \mathrm{~mm}$ thickness of silica gel (60 F-254), visualization was accomplished with $\mathrm{CeSO}_{4}$ or $10 \% \mathrm{H}_{2} \mathrm{SO}_{4} / \mathrm{EtOH}$ and subsequent charring over hot plate. Column chromatography was performed using silica gel (60-120), (100-200) and (230-400). All the products were characterized by ${ }^{1} \mathrm{H},{ }^{13} \mathrm{C}$, DEPT pulse sequence, Heteronuclear Single Quantum Correlation (HSQC), IR, MS (ESI), HRMS (EI) and HRMS (DART). All NMR spectra were recorded with spectrometers at 200, 300, $\left({ }^{1} \mathrm{H}\right)$ and $50,75, \mathrm{MHz}\left({ }^{13} \mathrm{C}\right)$. Experiments were 
recorded in $\mathrm{CDCl}_{3}$ at $25{ }^{\circ} \mathrm{C}$. Chemical shifts are given on the $\delta$ scale. Optical rotations were determined using a $1 \mathrm{dm}$ cell in chloroform as solvent; concentrations mentioned are in $\mathrm{g} / 100$ $\mathrm{mL}$.

General procedure for the synthesis of tetrahydrofuranyl 1,2,3-triazolyl $C$-nucleoside analogues. To a vigorously stirred solution of azide $(0.5 \mathrm{mmol})$ in tert-butyl alcohol $(5 \mathrm{~mL})$ was added acetylene $(0.6 \mathrm{mmol})$ and the reaction was initiated by the addition of a solution of $\mathrm{CuSO}_{4} .5 \mathrm{H}_{2} \mathrm{O}(0.1 \mathrm{mmol})$ and sodium ascorbate $(0.2 \mathrm{mmol})$ in distilled water. The colored suspension formed was stirred at the room temperature till the formation of triazole $(6-8 \mathrm{~h})$. After completion of the reaction, ice-cold water was added and the aqueous layer was extracted with $\mathrm{CHCl}_{3}(3 \times 15 \mathrm{~mL})$. The combined organic extracts were dried evaporated and purified by column chromatography to afford the pure 1,2,3-triazolyl $C$-nucleoside analogues.

Compound 14. Oil, 62\% yield, Eluent for column chromatography: $\mathrm{MeOH} / \mathrm{CHCl}_{3}(1 / 49$, v/v) $[\alpha]_{\mathrm{D}}{ }^{30}+33.3^{\circ}\left(c 0.30, \mathrm{CHCl}_{3}\right), R_{f} 0.32$ (3/2 EtOAc/hexane). IR (neat): $3428,3021,2929$, 2364, 1593, 1478,1216. ${ }^{1} \mathrm{H}$ NMR (300 MHz, $\left.\mathrm{CDCl}_{3}\right): \delta 1.39$ (s, 3H, $\left.\mathrm{CH}_{3}\right), 1.43\left(\mathrm{~s}, 3 \mathrm{H}, \mathrm{CH}_{3}\right)$, 2.84 (brs, $1 \mathrm{H}), 3.90(\mathrm{dd}, J 3.6,9.7 \mathrm{~Hz}, 1 \mathrm{H}), 4.01(\mathrm{dd}, J 6.1,8.4 \mathrm{~Hz}, 1 \mathrm{H}), 4.12-4.21(\mathrm{~m}, 4 \mathrm{H})$, 4.35-4.46 (m, 5H), 4.66-4.77 (m, 4H), $5.04(\mathrm{~d}, J 5.6 \mathrm{~Hz}, 1 \mathrm{H}), 5.11-5.14(\mathrm{~m}, 1 \mathrm{H}), 7.32-7.36$ $(\mathrm{m}, 10 \mathrm{H}, \mathrm{ArH}), 7.44\left(\mathrm{~s}, 1 \mathrm{H}\right.$, triazolyl H). ${ }^{13} \mathrm{C} \mathrm{NMR}\left(50 \mathrm{MHz}, \mathrm{CDCl}_{3}\right): \delta 25.8\left(\mathrm{CH}_{3}\right)$, 27.1 $\left(\mathrm{CH}_{3}\right), 66.0(\mathrm{CH}), 67.3\left(\mathrm{CH}_{2}\right), 70.7(\mathrm{CH}), 71.2\left(\mathrm{CH}_{2}\right), 73.4\left(\mathrm{CH}_{2}\right), 73.6\left(\mathrm{CH}_{2}\right), 73.6$ $(\mathrm{CH}), 73.8\left(\mathrm{CH}_{2}\right), 75.9(\mathrm{CH}), 82.3(\mathrm{CH}), 83.3(\mathrm{CH}), 83.9(\mathrm{CH}), 109.4(\mathrm{qC}) 121.5(\mathrm{CH}$ of triazole), 128.4 (ArC), 128.5 (ArC), 128.6 (ArC), 129.0 (ArC), 137.5 (ArqC), 137.6 (ArqC), 147.8 (qC of triazole). Mass (ESI-MS) $m / z$ 537, found $538[\mathrm{M}+\mathrm{H}]^{+}$.EI-HRMS $m / z$ : Calc. for $\mathrm{C}_{29} \mathrm{H}_{35} \mathrm{~N}_{3} \mathrm{O}_{7}[\mathrm{M}]^{+}$537.2475, measured 537.2490.

Compound 15. Oil, 72\% yield, Eluent for column chromatography: $\mathrm{MeOH} / \mathrm{CHCl}_{3}(1 / 49$, v/v) $[\alpha]_{\mathrm{D}}^{30}+25.2^{\circ}\left(c 0.30, \mathrm{CHCl}_{3}\right), R_{f} 0.32$ (3/2 EtOAc/hexane). IR (neat): 3430, 3020, 2925, 2360, 1600, 1376, 1216, 1067, 761. ${ }^{1} \mathrm{H}$ NMR $\left(300 \mathrm{MHz}, \mathrm{CDCl}_{3}\right): \delta 1.26\left(\mathrm{~s}, 3 \mathrm{H}, \mathrm{CH}_{3}\right), 1.34(\mathrm{~s}$, $\left.3 \mathrm{H}, \mathrm{CH}_{3}\right), 2.84(\mathrm{~S}, 1 \mathrm{H}), 3.76(\mathrm{dd}, J 5.9,8.5 \mathrm{~Hz}, 1 \mathrm{H}), 3.87-3.93(\mathrm{~m}, 2 \mathrm{H}), 4.08(\mathrm{dd}, J$ 6.1, 8.5 $\mathrm{Hz} 1 \mathrm{H}), 4.15-4.20(\mathrm{~m}, 2 \mathrm{H}), 4.23-4.35(\mathrm{~m}, 4 \mathrm{H}), 4.44(\mathrm{t}, J 5.3 \mathrm{~Hz}, 1 \mathrm{H}), 4.61-4.79(\mathrm{~m}, 4 \mathrm{H})$, $5.06(\mathrm{~d}, J 5.4 \mathrm{~Hz}, 1 \mathrm{H}), 5.27-5.28(\mathrm{~m}, 1 \mathrm{H}), 7.31-7.34(\mathrm{~m}, 10 \mathrm{H}, \mathrm{ArH}), 7.70(\mathrm{~S}, 1 \mathrm{H}$, triazolyl H). ${ }^{13} \mathrm{C}$ NMR $\left(75 \mathrm{MHz}, \mathrm{CDCl}_{3}\right): \delta 25.3\left(\mathrm{CH}_{3}\right), 26.5\left(\mathrm{CH}_{3}\right), 66.5(\mathrm{CH}), 66.6\left(\mathrm{CH}_{2}\right), 70.7$ $(\mathrm{CH}), 71.9\left(\mathrm{CH}_{2}\right), 72.9\left(\mathrm{CH}_{2}\right), 73.3\left(\mathrm{CH}_{2}\right), 73.6\left(\mathrm{CH}_{2}\right), 74.9(\mathrm{CH}), 76.1(\mathrm{CH}), 83.3(\mathrm{CH}), 85.5$ $(\mathrm{CH}), 85.9(\mathrm{CH}), 110.3(\mathrm{qC}) 121.4(\mathrm{CH}$ of triazole), $128.4(\mathrm{ArC}), 128.6(\mathrm{ArC}), 128.6(\mathrm{ArC})$, 128.6 (ArC), 129.0(ArC), 136.9 (ArqC), 137.6 (ArqC), 147.7 (qC of triazole). Mass (ESIMS) $\mathrm{m} / z$ 537, found $538[\mathrm{M}+\mathrm{H}]^{+}$. ESI-HRMS m/z: Calc. for $\mathrm{C}_{29} \mathrm{H}_{36} \mathrm{~N}_{3} \mathrm{O}_{7}[\mathrm{M}+\mathrm{H}]^{+}$ 538.2553, measured 538.2525.

Compound 16. Oil, 70\% yield, Eluent for column chromatography: $\mathrm{MeOH} / \mathrm{CHCl}_{3}(1 / 49$, v/v), $[\alpha]_{\mathrm{D}}^{29}-66.6^{\circ}\left(c 0.36, \mathrm{CHCl}_{3}\right), R_{f} 0.36$ (1/1 EtOAc/hexane). IR (KBr, $\left.\mathrm{cm}^{-1}\right): 3403,2988,2925$, 2364, 1725, 1457, 1218, 1068, 769. ${ }^{1} \mathrm{H}$ NMR $\left(200 \mathrm{MHz}, \mathrm{CDCl}_{3}\right): \delta 1.79$ (s, $\left.6 \mathrm{H}, 3 \mathrm{x} \mathrm{CH}_{2}\right), 2.23$ (s, 9H, $\left.3 \times \mathrm{CH}_{2}, 3 \times \mathrm{CH}\right), 2.82(\mathrm{~d}, J 4.4 \mathrm{~Hz}, 1 \mathrm{H}, \mathrm{OH}), 3.87(\mathrm{dd}, J 3.9,9.6 \mathrm{~Hz}, 1 \mathrm{H}), 4.17(\mathrm{dd}, J$ 4.9, $9.6 \mathrm{~Hz}, 1 \mathrm{H}), 4.32-4.36(\mathrm{~m}, 1 \mathrm{H}), 4.46(\mathrm{t}, J 5.4 \mathrm{~Hz}, 1 \mathrm{H}), 4.66(\mathrm{~d}, J 11.7 \mathrm{~Hz}, 1 \mathrm{H}), 4.77(\mathrm{~d}, J$ 
$11.7 \mathrm{~Hz}, 1 \mathrm{H}), 5.06(\mathrm{~d}, J 5.6 \mathrm{~Hz}, 1 \mathrm{H}), 7.32(\mathrm{brs}, 5 \mathrm{H}, \mathrm{ArH}), 7.52\left(\mathrm{~s}, 1 \mathrm{H}\right.$, triazolyl H) ${ }^{13} \mathrm{C}$ NMR $(50$ $\left.\mathrm{MHz}, \mathrm{CDCl}_{3}\right): \delta 29.9(3 \times \mathrm{CH}), 36.3\left(3 \times \mathrm{CH}_{2}\right), 43.4\left(3 \times \mathrm{CH}_{2}\right), 60.1$ (1C, qC adametane), 70.8, $(\mathrm{CH}), 73.3\left(\mathrm{CH}_{2}\right), 73.5\left(\mathrm{CH}_{2}\right), 76.4(\mathrm{CH}), 83.1(\mathrm{CH}), 118.9(\mathrm{CH}$ of triazole $), 128.5(\mathrm{ArC}), 128.5$ (ArC), 129.0 (ArC), 137.8 (ArqC), 146.5 (qC of triazole). Mass (ESI-MS) $m / z$ 395, found 396 $[\mathrm{M}+\mathrm{H}]^{+}$.ESI-HRMS: Calculated for $\mathrm{C}_{23} \mathrm{H}_{30} \mathrm{~N}_{3} \mathrm{O}_{3}[\mathrm{M}+\mathrm{H}]^{+}$396.2287, measured 396.2268.

Compound 18. Oil, 75\% yield, Eluent for column chromatography: $\mathrm{MeOH} / \mathrm{CHCl}_{3}(1 / 49$, v/v) $[\alpha]_{\mathrm{D}}{ }^{29}+184.3\left(c 0.70, \mathrm{CHCl}_{3}\right), R_{f} 0.32$ (3/2 EtOAc/hexane). IR (neat): 3483, 3021, 2931, 2367, 1217, 1070, 765. ${ }^{1} \mathrm{H}$ NMR (300 MHz, $\left.\mathrm{CDCl}_{3}\right): \delta 1.23\left(\mathrm{~s}, 3 \mathrm{H}, \mathrm{CH}_{3}\right), 1.31\left(\mathrm{~S}, 3 \mathrm{H}, \mathrm{CH}_{3}\right)$, 3.75 (dd, $J 5.9,8.5 \mathrm{~Hz}, 1 \mathrm{H}), 3.89(\mathrm{t}, J 4.8 \mathrm{~Hz}, 1 \mathrm{H}), 3.96-4.01(\mathrm{~m}, 5 \mathrm{H}), 4.17-4.34(\mathrm{~m}, 3 \mathrm{H})$, 4.40-4.50 (m, 3H), 4.55-4.74 (m, 2H), 5.21-5.24 (m, 2H), 7.13-7.17 (m, 2H, ArH), 7.26-7.33 $(\mathrm{m}, 8 \mathrm{H}, \mathrm{ArH}), 7.79\left(\mathrm{~s}, 1 \mathrm{H}\right.$, triazolyl H). ${ }^{13} \mathrm{C} \mathrm{NMR}\left(75 \mathrm{MHz}, \mathrm{CDCl}_{3}\right): \delta 25.3\left(\mathrm{CH}_{3}\right), 26.5$ $\left(\mathrm{CH}_{3}\right), 66.6(\mathrm{CH}), 66.7\left(\mathrm{CH}_{2}\right), 71.3(\mathrm{CH}), 71.8\left(\mathrm{CH}_{2}\right), 72.9\left(\mathrm{CH}_{2}\right), 73.3\left(\mathrm{CH}_{2}\right), 74.2\left(\mathrm{CH}_{2}\right)$, $74.9(\mathrm{CH}), 75.2(\mathrm{CH}), 79.5(\mathrm{CH}), 85.7(\mathrm{CH}), 85.9(\mathrm{CH}), 110.3(\mathrm{qC}), 128.0(\mathrm{ArC}), 128.3$ (ArC), 128.6 (ArC), 128.6 (ArC), 128.8 (ArC), 128.9 (ArC), 137.0 (ArqC), 137.6 (ArqC). Mass (ESI-MS) $m / z$ 537, found $538[\mathrm{M}+\mathrm{H}]^{+}$. ESI-HRMS $m / z$ : Calc. for $\mathrm{C}_{29} \mathrm{H}_{36} \mathrm{~N}_{3} \mathrm{O}_{7}[\mathrm{M}+\mathrm{H}]^{+}$ 538.2553, measured 538.2549.

Compound 19. Solid, mp 109-110 ${ }^{\circ} \mathrm{C}, 70 \%$ yield, Eluent for column chromatography: $\mathrm{MeOH} / \mathrm{CHCl}_{3}(1 / 49, \mathrm{v} / \mathrm{v})[\alpha]_{\mathrm{D}}^{29}+116.5^{\circ}\left(c 0.30, \mathrm{CHCl}_{3}\right), R_{f} 0.35$ (3/2 EtOAc/hexane). IR (neat): 3420, 3155, 3025, 2882, 2365, 1719, 1455, 1220, 1044, 742. ${ }^{1} \mathrm{H}$ NMR (300 MHz, $\left.\mathrm{CDCl}_{3}\right): \delta 1.37\left(\mathrm{~s}, 3 \mathrm{H}, \mathrm{CH}_{3}\right), 1.40\left(\mathrm{~s}, 3 \mathrm{H}, \mathrm{CH}_{3}\right), 3.83(\mathrm{~d}, J 7.8 \mathrm{~Hz}, 1 \mathrm{H}), 3.97-4.02(\mathrm{~m}, 3 \mathrm{H})$, 4.10-4.16 (m, 3H), 4.26-4.43 (m, 5H), $4.56(\mathrm{~d}, J 11.5,1 \mathrm{H}), 4.69(\mathrm{~S}, 2 \mathrm{H}), 5.08-5.12(\mathrm{~m}, 1 \mathrm{H})$, $5.21(\mathrm{~d}, J 6.2 \mathrm{~Hz}, 1 \mathrm{H}), 7.10-7.14(\mathrm{~m}, 2 \mathrm{H}, \operatorname{ArH}), 7.26-7.33(\mathrm{~m}, 8 \mathrm{H}, \operatorname{ArH}), 7.53(\mathrm{~s}, 1 \mathrm{H}$, triazolyl H). ${ }^{13} \mathrm{C}$ NMR $\left(50 \mathrm{MHz}, \mathrm{CDCl}_{3}\right): \delta 25.4\left(\mathrm{CH}_{3}\right), 26.7\left(\mathrm{CH}_{3}\right), 65.6(\mathrm{CH}), 66.9\left(\mathrm{CH}_{2}\right)$, $70.8(\mathrm{CH}), 71.0\left(\mathrm{CH}_{2}\right), 73.0\left(\mathrm{CH}_{2}\right), 73.1\left(\mathrm{CH}_{2}\right), 73.2(\mathrm{CH}), 73.8\left(\mathrm{CH}_{2}\right), 74.8(\mathrm{CH}), 79.2(\mathrm{CH})$, $81.8(\mathrm{CH}), 83.6(\mathrm{CH}), 109.0(\mathrm{qC}) 122.4(\mathrm{CH}$ of triazole), $127.6(\mathrm{ArC}), 127.9(\mathrm{ArC}), 128.0$ (ArC), 128.2 (ArC), 128.5 (ArC), 128.6 (ArC), 137.1 (ArqC), 137.2 (ArqC), Mass (ESI-MS) $\mathrm{m} / \mathrm{z}$ 537, found $538[\mathrm{M}+\mathrm{H}]^{+}, 560[\mathrm{M}+\mathrm{Na}]^{+}$.EI-HRMS m/z: Calc. for $\mathrm{C}_{29} \mathrm{H}_{36} \mathrm{~N}_{3} \mathrm{O}_{7}[\mathrm{M}+\mathrm{H}]^{+}$ 538.2553 , measured 538.2530.

Compound 20. Oil, 72\% yield, Eluent for column chromatography: $\mathrm{MeOH} / \mathrm{CHCl}_{3}(1 / 49, \mathrm{v} / \mathrm{v})$, $[\alpha]_{\mathrm{D}}^{29}+29.9\left(c 0.30, \mathrm{CHCl}_{3}\right), R_{f} 0.29$ (1/1 EtOAc/hexane). IR (KBr, cm $\left.{ }^{-1}\right): 3410,2921,2366$, $1813,1728,1653,1457,1375,1215,1067,752 .{ }^{1} \mathrm{H}$ NMR $\left(300 \mathrm{MHz}, \mathrm{CDCl}_{3}\right): \delta 1.79$ (s, 6H, $3 \mathrm{x}$ $\mathrm{CH}_{2}$ ), 2.21 (s, 6H, $3 \times \mathrm{CH}_{2}$ ), 2.26(s, 3H, $3 \times \mathrm{CH}$ ), 3.97-4.07 (m, $2 \mathrm{H}$ ), 4.17 (brs, $1 \mathrm{H}, \mathrm{OH}$ ), 4.27$4.37(\mathrm{~m}, 2 \mathrm{H}), 4.50-4.57(\mathrm{~m}, 2 \mathrm{H}), 5.24(\mathrm{~d}, J 5.6 \mathrm{~Hz}, 1 \mathrm{H}), 7.13$ (brs, $2 \mathrm{H}, \mathrm{ArH}), 7.28$ (brs, 3H, ArH), $7.65\left(\mathrm{~s}, 1 \mathrm{H}\right.$, triazolyl H). ${ }^{13} \mathrm{C} \mathrm{NMR}\left(50 \mathrm{MHz}, \mathrm{CDCl}_{3}\right): \delta 29.8(3 \times \mathrm{CH}), 36.3\left(3 \mathrm{x} \mathrm{CH}_{2}\right)$, $43.3\left(3 \times \mathrm{CH}_{2}\right), 60.0\left(1 \mathrm{C}, \mathrm{qC}\right.$ adametane), $71.4(\mathrm{CH}), 73.3\left(\mathrm{CH}_{2}\right), 74.3\left(\mathrm{CH}_{2}\right), 75.5(\mathrm{CH}), 79.5$ (CH), 128.1 (ArC), 128.3 (ArC), 128.7 (ArC), 137.7 (ArqC). Mass (ESI-MS) $\mathrm{m} / z$ 395, found 396 $[\mathrm{M}+\mathrm{H}]^{+}$and $418[\mathrm{M}+\mathrm{Na}]^{+}$. ESI-HRMS : Calculated for $\mathrm{C}_{23} \mathrm{H}_{30} \mathrm{~N}_{3} \mathrm{O}_{3}[\mathrm{M}+\mathrm{H}]^{+}$396.2287, measured 396.2272.

Compound 22. Oil, 68\% yield, Eluent for column chromatography: $\mathrm{MeOH} / \mathrm{CHCl}_{3}(1 / 49$, $\mathrm{v} / \mathrm{v})),[\alpha]_{\mathrm{D}}{ }^{30}+37^{\circ}\left(c \mathrm{0} .6, \mathrm{CHCl}_{3}\right), R_{f} 0.30$ (3/2 EtOAc/hexane). IR (neat): 3422, 3033, 2919 , 
2363, 2103, 1599, 1456, 1376, 1217, 1066, 732. ${ }^{1} \mathrm{H}$ NMR $\left(300 \mathrm{MHz}, \mathrm{CDCl}_{3}\right): \delta 1.39(\mathrm{~s}, 3 \mathrm{H}$, $\left.\mathrm{CH}_{3}\right), 1.47\left(\mathrm{~s}, 3 \mathrm{H}, \mathrm{CH}_{3}\right), 3.83(\mathrm{dd}, J 5.4,8.6 \mathrm{~Hz}, 1 \mathrm{H}), 4.03-4.22(\mathrm{~m}, 5 \mathrm{H}), 4.23-4.26(\mathrm{~m}, 2 \mathrm{H})$, 4.29- $4.35(\mathrm{~m}, 2 \mathrm{H}), 4.38-4.44(\mathrm{~m}, 3 \mathrm{H}), 4.59(\mathrm{~d}, J 11.7 \mathrm{~Hz}, 1 \mathrm{H}), 4.66(\mathrm{~d}, J 11.7 \mathrm{~Hz}, 1 \mathrm{H}), 4.92$ (brs, 1H), 5.05 (brs, 1H), $5.44(\mathrm{q}, J 6.0 \mathrm{~Hz}, 1 \mathrm{H}), 7.09-7.11(\mathrm{~m}, 2 \mathrm{H}), 7.28-7.32(\mathrm{~m}, 8 \mathrm{H}, \mathrm{ArH})$, $7.72\left(\mathrm{~s}, 1 \mathrm{H}\right.$, triazolyl H). ${ }^{13} \mathrm{C} \mathrm{NMR}\left(50 \mathrm{MHz}, \mathrm{CDCl}_{3}\right): \delta 25.2\left(\mathrm{CH}_{3}\right), 26.5\left(\mathrm{CH}_{3}\right), 66.5(\mathrm{CH})$, $66.6\left(\mathrm{CH}_{2}\right), 71.87\left(\mathrm{CH}_{2}\right), 72.3\left(\mathrm{CH}_{2}\right), 72.9\left(\mathrm{CH}_{2}\right), 74.9(\mathrm{CH}), 76.1(\mathrm{CH}), 76.2\left(\mathrm{CH}_{2}\right), 77.9$ $(\mathrm{CH}), 85.5(\mathrm{CH}), 85.9(\mathrm{CH}), 89.4(\mathrm{CH}), 110.3(\mathrm{qC}), 121.3(\mathrm{CH}$ of triazole), $128.1(\mathrm{ArC})$, 128.3 (ArC), 128.6 (ArC), 128.9 (ArC), 129.9 (ArC), 136.9 (ArqC), 137.9 (ArqC), 147.8 (qC of triazole). Mass (ESI-MS): $\mathrm{m} / z$ 537, found $538[\mathrm{M}+\mathrm{H}]^{+}$and $560[\mathrm{M}+\mathrm{Na}]^{+}$

Compound 23. Oil, 58\% yield, Eluent for column chromatography: $\mathrm{MeOH} / \mathrm{CHCl}_{3}(1 / 49$, $\mathrm{v} / \mathrm{v})),[\alpha]_{\mathrm{D}}^{30}+51.4^{\circ}\left(c 0.6, \mathrm{CHCl}_{3}\right), R_{f} 0.30$ (3/2 EtOAc/hexane). IR (neat): $3422,3033,2919$, 2363, 2103, 1599, 1456, 1376, 1217, 1066, 732. ${ }^{1} \mathrm{H}$ NMR (300 MHz, $\left.\mathrm{CDCl}_{3}\right): \delta 1.28(\mathrm{~s}, 3 \mathrm{H}$, $\left.\mathrm{CH}_{3}\right), 1.33\left(\mathrm{~s}, 3 \mathrm{H}, \mathrm{CH}_{3}\right), 3.78(\mathrm{dd}, J 5.7,8.6 \mathrm{~Hz}, 1 \mathrm{H}), 3.92(\mathrm{t}, J 4.7 \mathrm{~Hz}, 1 \mathrm{H}), 4.07-4.12(\mathrm{~m}$, $2 \mathrm{H}), 4.19-4.26(\mathrm{~m}, 4 \mathrm{H}), 4.31-4.36(\mathrm{~m}, 1 \mathrm{H}), 4.45$ (brs, $1 \mathrm{H}), 4.62-4.78(\mathrm{~m}, 5 \mathrm{H}), 5.05$ (brs, $1 \mathrm{H}), 5.26$ (brs, $1 \mathrm{H}), 7.31-7.35(\mathrm{~m}, 10 \mathrm{H}, \mathrm{ArH}), 7.74(\mathrm{~s}, 1 \mathrm{H}$, triazolyl $\mathrm{H}) .{ }^{13} \mathrm{C}$ NMR $(50 \mathrm{MHz}$, $\left.\mathrm{CDCl}_{3}\right): \delta 25.3\left(\mathrm{CH}_{3}\right), 26.5\left(\mathrm{CH}_{3}\right), 66.6(\mathrm{CH}), 66.6\left(\mathrm{CH}_{2}\right), 71.8\left(\mathrm{CH}_{2}\right), 72.3\left(\mathrm{CH}_{2}\right), 73.0$ $\left(\mathrm{CH}_{2}\right), 74.9(\mathrm{CH}), 76.1(\mathrm{CH}), 76.3\left(\mathrm{CH}_{2}\right), 77.9(\mathrm{CH}), 85.6(\mathrm{CH}), 86.0(\mathrm{CH}), 89.5(\mathrm{CH}), 110.3$ (qC), 121.3 ( $\mathrm{CH}$ of triazole), 128.1 (ArC), 128.3 (ArC), 128.7 (ArC), 128.9 (ArC), 129.0 (ArC), 137.0 (ArqC), 137.9 (ArqC), 147.8 (qC of triazole). Mass (ESI-MS) $\mathrm{m} / z$ 537, found $538[\mathrm{M}+\mathrm{H}]^{+}$. ESI-HRMS $\mathrm{m} / z$ : Calc. for $\mathrm{C}_{29} \mathrm{H}_{36} \mathrm{~N}_{3} \mathrm{O}_{7}[\mathrm{M}+\mathrm{H}]^{+}$538.2553, measured 538.2521 .

Compound 24. Solid, mp $112-114{ }^{\circ} \mathrm{C}$, $68 \%$ yield, Eluent for column chromatography: $\mathrm{MeOH} / \mathrm{CHCl}_{3}(1 / 49, \mathrm{v} / \mathrm{v}),[\alpha]_{\mathrm{D}}{ }^{28}+71.8^{\circ}\left(c 0.30, \mathrm{CHCl}_{3}\right), R_{f} 0.36$ (3/2 EtOAc/hexane). IR (neat): $3422,3033,2916,2363,2103,1599,1456,1376,1217,1066,738 .{ }^{1} \mathrm{H}$ NMR (300 $\left.\mathrm{MHz}, \mathrm{CDCl}_{3}\right): \delta 1.39\left(\mathrm{~s}, 3 \mathrm{H}, \mathrm{CH}_{3}\right), 1.43\left(\mathrm{~s}, 3 \mathrm{H}, \mathrm{CH}_{3}\right), 3.78-3.85(\mathrm{~m}, 1 \mathrm{H}), 3.99-4.24(\mathrm{~m}, 8 \mathrm{H})$, 4.35 (s, 1H), 4.38- 4.43 (m, 2H), 4.62-4.74 (m, 4H), 5.03 (brs, 1H), 5.10 (brs, 1H), 7.34-7.36 $(\mathrm{m}, 10 \mathrm{H}, \mathrm{ArH}), 7.47\left(\mathrm{~s}, 1 \mathrm{H}\right.$, triazolyl H). ${ }^{13} \mathrm{C} \mathrm{NMR}\left(75 \mathrm{MHz}, \mathrm{CDCl}_{3}\right): \delta 25.4\left(\mathrm{CH}_{3}\right), 26.7$ $\left(\mathrm{CH}_{3}\right), 65.6(\mathrm{CH}), 66.9\left(\mathrm{CH}_{2}\right), 70.7\left(\mathrm{CH}_{2}\right), 72.1\left(\mathrm{CH}_{2}\right), 73.2(\mathrm{CH}), 73.2\left(\mathrm{CH}_{2}\right), 75.8$ $(\mathrm{CH}), 77.6(\mathrm{CH}), 81.8(\mathrm{CH}), 83.4(\mathrm{CH}), 89.0(\mathrm{CH}), 109.0(\mathrm{qC}), 120.9(\mathrm{CH}$ of triazole $), 127.8$ (ArC), 127.9 (ArC), 128.0 (ArC), 128.2 (ArC), 128.5, (ArC), 128.6 (ArC), 137.1 (ArqC), 137.6 (ArqC), 147.5 (qC of triazole). Mass (ESI-MS) $m / z$ 537, found $538[\mathrm{M}+\mathrm{H}]^{+}$. ESIHRMS $m / z$ : Calc. for $\mathrm{C}_{29} \mathrm{H}_{36} \mathrm{~N}_{3} \mathrm{O}_{7}[\mathrm{M}+\mathrm{H}]^{+}$538.2553, measured 538.2533.

Compound 25. Oil, 78\% yield, eluent for column chromatography: $\mathrm{MeOH} / \mathrm{CHCl}_{3}(1 / 49$, v/v), $[\alpha]_{\mathrm{D}}^{30}+44.0^{\circ}\left(c 1.20, \mathrm{CHCl}_{3}\right), R_{f} 0.30\left(1 / 1 \mathrm{EtOAc} /\right.$ hexane). IR (Neat, $\left.\mathrm{cm}^{-1}\right): 3327,3008,2919$, 2857, 2361, 1726, 1598, 1454, 1218, 1099, 759. ${ }^{1} \mathrm{H}$ NMR $\left(200 \mathrm{MHz}, \mathrm{CDCl}_{3}\right): \delta 1.79$ (s, 6H, $3 \mathrm{x}$ $\mathrm{CH}_{2}$ ), 2.23 (brs, 9H, $3 \times \mathrm{CH}_{2}, 3 \times \mathrm{CH}$ ), $4.07(\mathrm{~d}, J 9.5 \mathrm{~Hz}, 1 \mathrm{H}), 4.19-4.28(\mathrm{~m}, 2 \mathrm{H}), 4.43$ (brs, $\left.1 \mathrm{H}\right)$, $4.65\left(\mathrm{~d}, J 11.9 \mathrm{~Hz}, 1 \mathrm{H}, \mathrm{CH}_{2} \mathrm{Ph}\right), 4.69\left(\mathrm{~d}, J 11.9 \mathrm{~Hz}, 1 \mathrm{H}, \mathrm{CH}_{2} \mathrm{Ph}\right), 5.06$ (s, $\left.1 \mathrm{H}\right), 5.38$ (brs, $\left.1 \mathrm{H}\right) 7.33$ (brs, $5 \mathrm{H}, \mathrm{ArH}), 7.57\left(\mathrm{~S}, 1 \mathrm{H}\right.$, triazolyl H). ${ }^{13} \mathrm{C} \mathrm{NMR}\left(50 \mathrm{MHz}, \mathrm{CDCl}_{3}\right): \delta 29.8(3 \mathrm{x} \mathrm{CH}), 36.2(3 \mathrm{x}$ $\left.\mathrm{CH}_{2}\right), 43.3\left(3 \times \mathrm{CH}_{2}\right), 60.4\left(1 \mathrm{C}, \mathrm{qC}\right.$ adametane) $72.3\left(\mathrm{CH}_{2}\right), 76.1(\mathrm{CH}), 76.4\left(\mathrm{CH}_{2}\right), 77.9(\mathrm{CH})$, $89.4(\mathrm{CH}), 118.9(\mathrm{CH}$ of triazole), $128.2(\mathrm{ArC}), 128.3(\mathrm{ArC}), 128.9(\mathrm{ArC}), 138.1(\mathrm{ArqC}), 146.3$ 
(qC of triazole). Mass (ESI-MS) $m / z$ 395, found $396[\mathrm{M}+\mathrm{H}]^{+}$and $418[\mathrm{M}+\mathrm{Na}]^{+}$. ESI-HRMS $m / z$ : Calc. for $\mathrm{C}_{23} \mathrm{H}_{30} \mathrm{~N}_{3} \mathrm{O}_{3}[\mathrm{M}+\mathrm{H}]^{+}$396.2287, measured 396.2262.

Compound 27. Solid, mp: $162-164{ }^{\circ} \mathrm{C}, 70 \%$ yield, Eluent for column chromatography: $\mathrm{MeOH} / \mathrm{CHCl}_{3}(1 / 49, \mathrm{v} / \mathrm{v}),[\alpha]_{\mathrm{D}}{ }^{29}-8.8^{\circ}\left(c 0.30, \mathrm{CHCl}_{3}\right), R_{f} 0.33(1 / 1 \mathrm{EtOAc} / \mathrm{hexane})$. IR $\left(\mathrm{KBr}, \mathrm{cm}^{-}\right.$ $\left.{ }^{1}\right): 3205,2915,2363,1813,1725,1662,1592,1446,1296,1225,1130,727 .{ }^{1} \mathrm{H}$ NMR (200 MHz, $\left.\mathrm{CDCl}_{3}\right): \delta 1.76\left(\mathrm{~s}, 6 \mathrm{H}, 3 \times \mathrm{CH}_{2}\right), 2.16\left(\mathrm{~s}, 6 \mathrm{H}, 3 \times \mathrm{CH}_{2}\right.$ ), $2.22(\mathrm{~s}, 3 \mathrm{H}, 3 \times \mathrm{CH}$ ), 3.49 (brs, 1H, $\mathrm{OH}), 3.85(\mathrm{~d}, J 9.8 \mathrm{~Hz}, 1 \mathrm{H}), 4.06(\mathrm{~s}, 1 \mathrm{H}), 4.18-4.33(\mathrm{~m}, 2 \mathrm{H}), 4.47-4.57(\mathrm{~m}, 2 \mathrm{H}), 5.48(\mathrm{~s}, 1 \mathrm{H})$, 7.07 (brs, 2H, ArH), 7.23 (brs, 3H, ArH), 7.68 (s, 1H, triazolyl H). ${ }^{13} \mathrm{C} \mathrm{NMR} \mathrm{(50} \mathrm{MHz,} \mathrm{CDCl}{ }_{3}$ ): $\delta 29.8(3 \times \mathrm{CH}), 36.3\left(3 \times \mathrm{CH}_{2}\right), 43.3\left(3 \times \mathrm{CH}_{2}\right), 59.9(1 \mathrm{C}, \mathrm{qC}$ adametane $) 72.6\left(\mathrm{CH}_{2}\right), 74.3$ $\left(\mathrm{CH}_{2}\right), 75.9(\mathrm{CH}), 76.8(\mathrm{CH}), 85.7(\mathrm{CH}), 120.6(\mathrm{CH}$ of triazole $), 127.7(\mathrm{ArC}), 128.0(\mathrm{ArC}), 128.6$ $(\mathrm{ArC}), 138.3$ (ArqC), 144.0 (qC of triazole). Mass (ESI-MS) $\mathrm{m} / z, 395$, found $396[\mathrm{M}+\mathrm{H}]^{+}$and $418[\mathrm{M}+\mathrm{Na}]^{+}$. ESI-HRMS : Calculated for $\mathrm{C}_{23} \mathrm{H}_{30} \mathrm{~N}_{3} \mathrm{O}_{3}[\mathrm{M}+\mathrm{H}]^{+}$396.2287, measured 396.2265 .

\section{Acknowledgements}

The authors are thankful to Sophisticated Analytical Instrument Facility (SAIF), CDRI Lucknow, India for providing spectral data and Mr. Anoop K. Pandey for technical assistance. DST grant (No. SR/SI/OC-17/2010) to support the work is gratefully acknowledged. CDRI communication no 8564 .

\section{References}

1. Štambaský, J.; Hocek, M.; Kocovsky, P. Chem. Rev. 2009, 109, 6729-6764. http://dx.doi.org/10.1021/cr9002165 PMid:19761208

2. Ferrero, M.; Gotor, V. Chem. Rev. 2000, 100, 4319-4347. http://dx.doi.org/10.1021/cr000446y PMid:11749350

3. Adamo, M. F. A.; Adlington, R. M.; Baldwin, J. E.; Day, A. L. Tetrahedron 2004, 60, 841849. http://dx.doi.org/10.1016/j.tet.2003.11.055

4. Qu, F.; Hang, J. H.; Du, J.; Newton, M. G.; Chu, C. K. Tetrahedron 1999, 55, 9073-9088. http://dx.doi.org/10.1016/S0040-4020(99)00499-8

5. Urban, M.; Pohl, R.; Klepetarova, B.; Hocek, M. J. Org. Chem. 2006, 71, 7322-7328. http://dx.doi.org/10.1021/jo061080d PMid:16958526

6. Rashad, A. E.; Shamroukh, A. H.; Hegab, M. I.; Awad, H. M. Acta Chim. Slov. 2005, 52, 429-434 and references therein. 
7. Ramasamy, K. S.; Bandaru, R.; Averett, D. J. Org. Chem. 2000, 65, 5849-5851. http://dx.doi.org/10.1021/jo000460a

8. Trost, B. M.; Kallander, L. S. J. Org. Chem. 1999, 64, 5427-5435. http://dx.doi.org/10.1021/jo990195x

9. Sallam, M. A. E.; Luis, F. F.; Cassady, J. M. Nucleos. Nucleot. Nucl. 2000, 19, 941-954. http://dx.doi.org/10.1080/15257770008033034 PMid:10893713

10. Shaban, M. A. E.; Nasr, A. Z. Adv. Heterocycl. Chem. 1997, 68, 223-432.

11. Qu, F.; Hong, J. H.; Du, J.; Newton, M. G.; Chu, C. K. Tetrahedron 1999, 55, 9073-9088. http://dx.doi.org/10.1016/S0040-4020(99)00499-8

12. Heinrich, D.; Wagner, T.; Diederichsen, U. Org. Lett. 2007, 9, 5311-5314. http://dx.doi.org/10.1021/ol7025334 PMid:17999513

13. Kharb, R.; Sharma, P. C.; Yar, M. S. J. Enzyme Inhib. Med. Chem. 2011, 26, 1-21 and references cited therein. http://dx.doi.org/10.3109/14756360903524304 PMid:20583859

14. Agalave, S. G.; Maujan, S. R.; Pore, V. S. Chem. Asian J. 2011, 6, 2696-2718 and references cited therein http://dx.doi.org/10.1002/asia.201100432 PMid:21954075

15. Kumar, A.; Ahmad, I.; Chhikara, B. S.; Tiwari, R.; Mandal, D.; Parang, K. Bioorg. Med. Chem. Lett. 2011, 21, 1342-1346. http://dx.doi.org/10.1016/j.bmcl.2011.01.047 PMid:21300544

16. Pereira, D.; Fernandes, P. Bioorg. Med. Chem. Lett. 2011, 21, 510-513. http://dx.doi.org/10.1016/j.bmcl.2010.10.091 PMid:21084187

17. Holla, B. S.; Mahalinga, M.; Karthikeyan, M. S.; Poojary, B.; Akberali, P. M.; Kumari, N. S. Eur. J. Med. Chem. 2005, 40, 1173-1178. http://dx.doi.org/10.1016/j.ejmech.2005.02.013 PMid:15979767

18. Genin, M. J.; Allwine, D. A.; Anderson, D. J.; Barbachyn, M. R.; Emmert, D. E.; Garmon, S. A.; Graber, D. R.; Grega, K. C.; Hester, J. B.; Hutchinson, D. K.; Morris, J.; Reischer, R. J.; Ford, C. W.; Zurenko, G. E.; Hamel, J. C.; Schaadt, R. D.; Stapert, D.; Yagi, B. H. J. Med. Chem. 2000, 43, 953-970. http://dx.doi.org/10.1021/jm990373e

19. Manfredini, S.; Vicentini, C. B.; Manfrini, M.; Bianchi, N.; Rutigliano, C.; Mischiati, C.; Gambari, R. Bioorg. Med. Chem. 2000, 8, 2343-2346. http://dx.doi.org/10.1016/S0968-0896(00)00160-7

20. Nájera, C.; Mansano, J. M. Org. Biomol. Chem. 2009, 7, 4567-4581. http://dx.doi.org/10.1039/b913066g PMid:19865689

21. Pradere, U.; Roy, V.; McBrayer, T. R.; Schinazi, R. F.; Agrofoglio, L. A. Tetrahedron 2008, 64, 9044-9051. http://dx.doi.org/10.1016/j.tet.2008.07.007 
22. Alvarez, R.; Velázquez, S.; San-Felix, A.; Aquaro, S.; De Clercq, E.; Perno, C. F.; Karlsson, A.; Balzarini, J.; Camarasa, M. J. J. Med. Chem. 1994, 37, 4185-4194. http://dx.doi.org/10.1021/jm00050a015

23. Haebich, D.; Barth, W.; Rosner, M. Heterocycles 1989, 29, 2083-2088; http://dx.doi.org/10.3987/COM-89-5171

24. Pérez-Castro, I.; Caama-o, O.; Fernández, F.; García, M. D.; López, C.; Clercq, E. D. Org. Biomol. Chem. 2007, 5, 3805-3813. http://dx.doi.org/10.1039/b710348d PMid:18004460

25. Pérez-Castro, I.; Caama-o, O.; Fernández, F.; García, M. D.; López, C.; Clercq, E. D. Arkivoc 2010, (iii), 152-168.

26. Cho, J. H.; Bernard, D. L.; Sidwell, R. W.; Kern, E. R.; Chu, C. K. J. Med. Chem. 2006, 49, 1140-1148.

http://dx.doi.org/10.1021/jm0509750

PMid:16451078

27. Joubert, N.; Schinazi, R. F.; Agrofoglio, L. A. Tetrahedron 2005, 61, 11744-11750. http://dx.doi.org/10.1016/j.tet.2005.09.034

28. Saito, Y.; Escuret, V.; Durantel, D.; Zoulim, F.; Schinazi, R. F.; Agrofoglio, L. A. Bioorg. Med. Chem. 2003, 11, 3633-3639. http://dx.doi.org/10.1016/S0968-0896(03)00349-3

29. Sallam, M. A. E.; Louis, F. F. Chirality 2004, 16, 331-335. http://dx.doi.org/10.1002/chir.20033 PMid:15069664

30. Sallam, M. A. E.; Hegazy, E. I. A. Carbohydr. Res. 1982, 102, 197-206. http://dx.doi.org/10.1016/S0008-6215(00)88062-3

31. Sallam, M. A. E. Carbohydr. Res. 1982, 106, 71-82. http://dx.doi.org/10.1016/S0008-6215(00)80733-8

32. Sallam, M. A. E.; Hegazy, E. I. A. Carbohydr. Res. 1981, 95, 177-188. http://dx.doi.org/10.1016/S0008-6215(00)85574-3

33. Sallam, M. A. E. Carbohydr. Res. 1981, 91, 139-148. http://dx.doi.org/10.1016/S0008-6215(00)86026-7

34. Sallam, M. A. E. Carbohydr. Res. 1980, 85, 93-105. http://dx.doi.org/10.1016/S0008-6215(00)84566-8

35. Kaliappan, K. P.; Kalanidhi, P.; Mahapatra, S. Synlett 2009, 2162-2166. http://dx.doi.org/10.1055/s-0029-1217570

36. Youcef, R. A.; Santos, M. D.; Roussel, S.; Baltaze, J.-P.; Lubin-Germain, N.; Uziel, J. J. Org. Chem. 2009, 74, 4318-4323.

http://dx.doi.org/10.1021/jo900594x

PMid:19438214

37. Adamo, M. F. A.; Pergoli, R. Org. Lett. 2007, 9, 4443-4446. http://dx.doi.org/10.1021/ol701794u PMid:17910462

38. Meldal, M.; Tornøe, C. W. Chem. Rev. 2008, 108, 2952-3015. http://dx.doi.org/10.1021/cr0783479 PMid:18698735 
39. Tornøe, C. W.; Christensen, C.; Meldal, M. J. Org. Chem. 2002, 67, 3057-3064. http://dx.doi.org/10.1021/jo011148j PMid:11975567

40. Rostovtsev, V. V.; Green, L. G.; Fokin, V. V.; Sharpless, K. B. Angew. Chem. Int. Ed. 2002, 41, 2596-2599. . http://dx.doi.org/10.1002/1521-3773(20020715)41:14<2596::AID-ANIE2596>3.0.CO;2-4

41. Kushwaha, D.; Dwivedi, P.; Kuanar, S. K.; Tiwari, V. K Curr. Org. Synth. 2013, 10, 90-135. http://dx.doi.org/10.2174/157017913804811004

42. Huisgen, R. Pure Appl. Chem. 1989, 61, 613-628. http://dx.doi.org/10.1351/pac198961040613

43. Huisgen, R.; Szeimies, G.; Moebius, L. Chem. Ber. 1967, 100, 2494-2507. http://dx.doi.org/10.1002/cber.19671000806

44. Sagar, R.; Reddy, L. V. R.; Saquib, M.; Kumar, B.; Shaw, A. K. Tetrahedron: Asymmetry 2006, 17, 3294-3299. http://dx.doi.org/10.1016/j.tetasy.2006.12.010

45. Reddy, L.V.R.; Kumar, V.; Sagar, R.; Shaw, A. K. Chem. Rev. 2013, 113, 3605-3631. http://dx.doi.org/10.1021/cr200016m PMid:23419115

46. Reddy, P. V.; Bajpai, V.; Kumar, B.; Shaw, A. K. Eur. J. Org. Chem. 2011, 75, 1575-1586.

47. Reddy, P. V.; Reddy, L. V. R.; Kumar, B.; Kumar, R.; Maulik, P. R.; Shaw A. K. Tetrahedron 2008, 64, 2153-2159. http://dx.doi.org/10.1016/j.tet.2007.12.032

48. Wanka, L.; Iqbal, K.; Schreiner, P. R. Chem. Rev. 2013, 113, 3516-3604. http://dx.doi.org/10.1021/cr100264t PMid:23432396

49. Liu, J.; Obando, D.; Liao, V.; Lifa, T.; Codd, R. Eur. J. Med. Chem. 2011, 46, 1949-1963. http://dx.doi.org/10.1016/j.ejmech.2011.01.047 PMid:21354674

50. Orzeszko, B.; Laudy, A. E.; Starosciak, B. J.; Orzeszko, A.; Kazimierczuk, Z. Acta Polo. Pharm. 2004, 61, 455-460.

51. Andrzej, O.; Beata, K.; Orzeszko, G.; Starosciak, B. J. Farmaco Il. 2000, 55, 619-623. http://dx.doi.org/10.1016/S0014-827X(00)00075-6 